\title{
Die EZB vor Gericht? Teil I: Wer klagen könnte, und wogegen
}

VB verfassungsblog.de/die-ezb-vor-gericht-teil-wer-klagen-knnte-und-wogegen/

Franz C. Mayer Mo 10 Sep 2012

Mo 10 Sep

2012

Jetzt also auch die Europäische Zentralbank. Die jüngste Ankündigung der EZB erneut Staatsanleihen von EuroStaaten ankaufen zu wollen hat sofort eine Debatte ausgelöst, ob die EZB das denn überhaupt darf oder nicht und ob man nicht die Gerichte einschalten müsste. Das zu fragen ist natürlich nicht verboten. Aber es setzt sich fort, was die deutsche Europadebatte seit geraumer Zeit prägt: Politiker argumentieren juristisch, mutieren zu HobbyEuroparechtlern und Freizeit-Verfassungsexperten. Es gibt auch schon wieder den obligatorischen aussichtslosen - Gauweiler-Antrag an das BVerfG zum gerade akuten EU-Problem, er erhält ja auch als eine Art Poweruser des Verfassungsgerichts einen lukrativen Vielkläger-Bonus. Bei solchen und ähnlichen Manövern notorischer Europaskeptiker gerät die Frage, was eigentlich politisch gewollt, vertretbar, nützlich oder geboten ist und was die Alternative des Nichthandelns bedeutet, immer mehr in den Hintergrund.

Nun denn - wie sieht es aus mit dem Handeln der EZB und der Vereinbarkeit mit dem Europarecht? Und könnte man die nach allgemeiner Wahrnehmung unabhängige EZB überhaupt verklagen?

I. Es erscheint vielleicht verblüffend, aber Klagen gegen die EZB sind im Europarecht ganz selbstverständlich vorgesehen. Gegen Handlungen der EZB kommt die Nichtigkeitsklage nach Art. 263 AEUV in Betracht. Danach überwacht der EuGH auch die Rechtmäßigkeit der Handlungen der EZB. Sekundär- und Primärmarktankäufe oder auch Kreditvergaben an einen mit Bankenlizenz ausgestatteten ESM könnten solche Handlungen sein.

II. Klagen können die Mitgliedstaaten, aber auch Rat, Europäische Kommission und EU-Parlament sowie, soweit es um die Wahrung ihrer Rechte geht, die EZB selbst, der Rechnungshof und der Ausschuss der Regionen. Unter bestimmten Bedingungen kann zudem jede natürliche oder juristische Person klagen. Dies wird durch den EuGH traditionell äußerst restriktiv gehandhabt, im Unterschied zum BVerfG, das mit einer gewagten richterrechtlichen Konstruktion aus dem Wahlrecht ein individuelles Klagerecht gegen EU-Verträge herleitet und diese wacklige Konstruktion höher und noch höher baut. Vor dem EuGH dagegen muss man individuell und unmittelbar betroffen sein, um klagen zu können. Manche meinen, dass unter diesen Voraussetzungen auch eine nationale Zentralbank klagen könnte. Das sollte man Herrn Weidmann wahrscheinlich besser nicht verraten.

III. Wogegen richtet sich die Klage? Es muss sich um Rechtsakte handeln, die verbindliche Wirkung nach außen entfalten, also nicht bloße Realakte. Hier wird es schon schwierig mit der Beanstandung der Sekundärmarktankäufe. Nehmen wir das ab 2010 durchgeführte Ankaufprogramm von Staatsanleihen auf dem Sekundärmarkt. Es beruht auf dem Beschluss EZB/2010/5 vom 14.5.2010 (Details nachzulesen bei Herrmann, EuZW 2010, 645), der die nationalen Zentralbanken ermächtigt, Staatsanleihen der Euro-Mitgliedstaaten auf dem Sekundärmarkt anzukaufen. Ist das eine Außenwirkung? Fraglich. Denn das Europäische System der Zentralbanken (ESZB) besteht aus der EZB und den nationalen Zentralbanken, so dass der Beschluss eigentlich nur ESZB-interne Wirkung hat.

IV. Nicht zu vergessen: Es gibt eine Klagefrist. Sie beträgt 2 Monate.

V. Wie verträgt sich die Möglichkeit, die EZB zu verklagen, mit deren Unabhängigkeit? Die schreibt Art. 130 AEUV (ex-Artikel 108 EGV) explizit vor: 
nationale Zentralbank noch ein Mitglied ihrer Beschlussorgane Weisungen von Organen, Einrichtungen oder sonstigen Stellen der Union, Regierungen der Mitgliedstaaten oder anderen Stellen einholen oder entgegennehmen. Die Organe, Einrichtungen oder sonstigen Stellen der Union sowie die Regierungen der Mitgliedstaaten verpflichten sich, diesen Grundsatz zu beachten und nicht zu versuchen, die Mitglieder der Beschlussorgane der Europäischen Zentralbank oder der nationalen Zentralbanken bei der Wahrnehmung ihrer Aufgaben zu beeinflussen."

Die EZB ist als eine "countermajoritarian institution" angelegt. Die EZB soll gerade nicht durch einen Mehrheitswillen kontrolliert werden. Wie die Verfassungsgerichtsbarkeit soll sie dem demokratisch geformten Mehrheitswillen (des Parlaments) nicht unterworfen sein - ein schwer aufzulösendes demokratietheoretisches Problem.

Ist es Beeinflussung, wenn Mitgliedstaaten anfangen, die EZB vor den EuGH zu zerren? Das könnte womöglich zu einem rechtlichen Problem in umgekehrter Richtung werden: Am Ende verklagt noch jemand (z.B. die Europäische Kommission, der Rat, das EP oder die EZB selbst) zum Beispiel Deutschland, weil Forderungen staatlicher Stellen (es gab da kürzlich recht harsche Forderungen des hessischen Justizministers an die EZB) gegen Art. 130 AEUV verstoßen ...

VI. Unabhängigkeit heißt aber nicht, dass man tun kann, was man will. Der EuGH hat im Juli 2003 klargestellt, dass die EZB der Kontrolle durch den EuGH unterliegt. In dem Urteil heißt es wörtlich (Rs. C-11/00, u.a. Leitsatz 6):

„Dagegen hat diese Zuerkennung einer solchen Unabhängigkeit nicht zur Folge, dass die
Europäische Zentralbank völlig von der Europäischen Gemeinschaft gesondert und von jeder
Bestimmung des Gemeinschaftsrechts ausgenommen wäre. Nichts erlaubt es, von vornherein
auszuschließen, dass der Gemeinschaftsgesetzgeber gemäß den Befugnissen, über die er nach
dem EG-Vertrag verfügt, und unter den im EG-Vertrag vorgesehenen Bedingungen normative
Maßnahmen erlässt, die Geltung gegenüber der Europäischen Zentralbank beanspruchen können."

Klingt an sich unmissverständlich: Die EZB steht ganz eindeutig nicht außerhalb des Rechts, noch nicht einmal außerhalb des Sekundärrechts. Das heißt aber noch nicht, dass der EuGH die EZB auch immer und stets sehr eng kontrollieren würde. Viel wahrscheinlicher ist er ihr einen weiten Spielraum einräumt, um zu entscheiden, was zu tun ist, um die Währung stabil zu halten. Das wäre jedenfalls eine sinnvolle Rechtsprechung im Sinne richterlicher Zurückhaltung im Wissen um die Grenzen dessen, was ein Gericht in der Euro-Krise leisten kann. Dass der EuGH die Lage besser überblickt als die EZB, ist jedenfalls nicht wahrscheinlich. Das gilt übrigens auch für das BVerfG im Verhältnis zu den anderen Akteuren in der Euro-Krise.

Teil 2 folgt! 


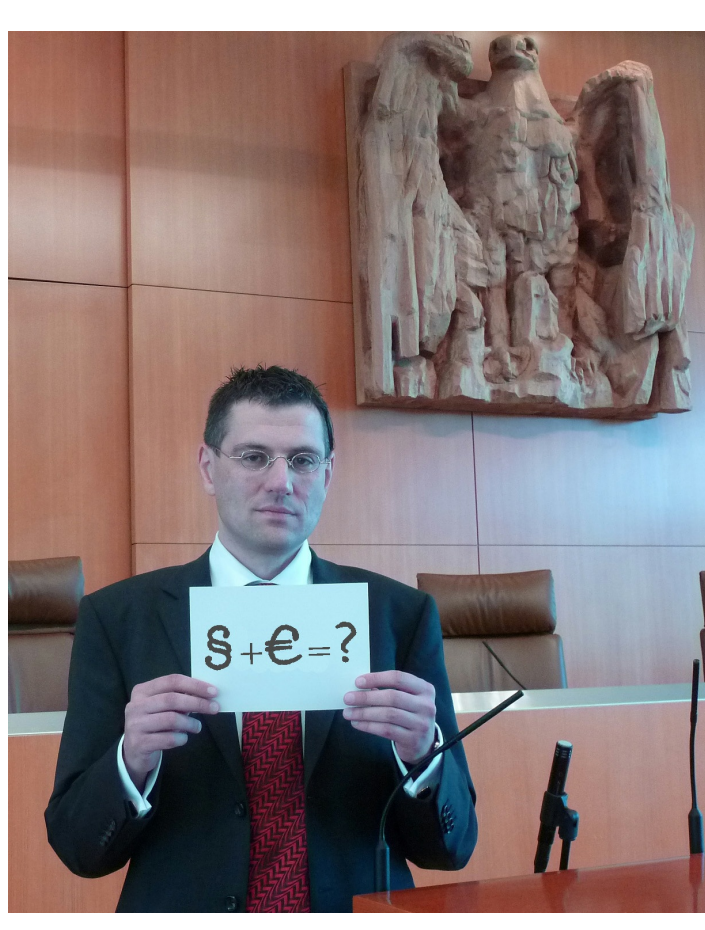

Jetzt also auch die Europäische Zentralbank. Die jüngste Ankündigung der EZB erneut Staatsanleihen von Euro-Staaten ankaufen zu wollen hat sofort eine Debatte ausgelöst, ob die EZB das denn überhaupt darf oder nicht und ob man nicht die Gerichte einschalten müsste. Das zu fragen ist natürlich nicht verboten. Aber es setzt sich fort, was die deutsche Europadebatte seit geraumer Zeit prägt: Politiker argumentieren juristisch, mutieren zu HobbyEuroparechtlern und Freizeit-Verfassungsexperten. Es gibt auch schon wieder den obligatorischen - aussichtslosen - GauweilerAntrag an das BVerfG zum gerade akuten EU-Problem, er erhält ja auch als eine Art Poweruser des Verfassungsgerichts einen lukrativen Vielkläger-Bonus. Bei solchen und ähnlichen Manövern notorischer Europaskeptiker gerät die Frage, was eigentlich politisch gewollt, vertretbar, nützlich oder geboten ist und was die Alternative des Nichthandelns bedeutet, immer mehr in den Hintergrund.

Nun denn - wie sieht es aus mit dem Handeln der EZB und der Vereinbarkeit mit dem Europarecht? Und könnte man die nach allgemeiner Wahrnehmung unabhängige EZB überhaupt verklagen?

I. Es erscheint vielleicht verblüffend, aber Klagen gegen die EZB sind im Europarecht ganz selbstverständlich vorgesehen. Gegen Handlungen der EZB kommt die Nichtigkeitsklage nach Art. 263 AEUV in Betracht. Danach überwacht der EuGH auch die Rechtmäßigkeit der Handlungen der EZB. Sekundär- und Primärmarktankäufe oder auch Kreditvergaben an einen mit Bankenlizenz ausgestatteten ESM könnten solche Handlungen sein.

II. Klagen können die Mitgliedstaaten, aber auch Rat, Europäische Kommission und EU-Parlament sowie, soweit es um die Wahrung ihrer Rechte geht, die EZB selbst, der Rechnungshof und der Ausschuss der Regionen. Unter bestimmten Bedingungen kann zudem jede natürliche oder juristische Person klagen. Dies wird durch den EuGH traditionell äußerst restriktiv gehandhabt, im Unterschied zum BVerfG, das mit einer gewagten richterrechtlichen Konstruktion aus dem Wahlrecht ein individuelles Klagerecht gegen EU-Verträge herleitet und diese wacklige Konstruktion höher und noch höher baut. Vor dem EuGH dagegen muss man individuell und unmittelbar betroffen sein, um klagen zu können. Manche meinen, dass unter diesen Voraussetzungen auch eine nationale Zentralbank klagen könnte. Das sollte man Herrn Weidmann wahrscheinlich besser nicht verraten.

III. Wogegen richtet sich die Klage? Es muss sich um Rechtsakte handeln, die verbindliche Wirkung nach außen entfalten, also nicht bloße Realakte. Hier wird es schon schwierig mit der Beanstandung der Sekundärmarktankäufe. Nehmen wir das ab 2010 durchgeführte Ankaufprogramm von Staatsanleihen auf dem Sekundärmarkt. Es beruht auf dem Beschluss EZB/2010/5 vom 14.5.2010 (Details nachzulesen bei Herrmann, EuZW 2010, 645), der die nationalen Zentralbanken ermächtigt, Staatsanleihen der Euro-Mitgliedstaaten auf dem Sekundärmarkt anzukaufen. Ist das eine Außenwirkung? Fraglich. Denn das Europäische System der Zentralbanken (ESZB) besteht aus der EZB und den nationalen Zentralbanken, so dass der Beschluss eigentlich nur ESZB-interne Wirkung hat.

IV. Nicht zu vergessen: Es gibt eine Klagefrist. Sie beträgt 2 Monate.

V. Wie verträgt sich die Möglichkeit, die EZB zu verklagen, mit deren Unabhängigkeit? Die schreibt Art. 130 AEUV (ex-Artikel 108 EGV) explizit vor: 
nationale Zentralbank noch ein Mitglied ihrer Beschlussorgane Weisungen von Organen, Einrichtungen oder sonstigen Stellen der Union, Regierungen der Mitgliedstaaten oder anderen Stellen einholen oder entgegennehmen. Die Organe, Einrichtungen oder sonstigen Stellen der Union sowie die Regierungen der Mitgliedstaaten verpflichten sich, diesen Grundsatz zu beachten und nicht zu versuchen, die Mitglieder der Beschlussorgane der Europäischen Zentralbank oder der nationalen Zentralbanken bei der Wahrnehmung ihrer Aufgaben zu beeinflussen."

Die EZB ist als eine "countermajoritarian institution" angelegt. Die EZB soll gerade nicht durch einen Mehrheitswillen kontrolliert werden. Wie die Verfassungsgerichtsbarkeit soll sie dem demokratisch geformten Mehrheitswillen (des Parlaments) nicht unterworfen sein - ein schwer aufzulösendes demokratietheoretisches Problem.

Ist es Beeinflussung, wenn Mitgliedstaaten anfangen, die EZB vor den EuGH zu zerren? Das könnte womöglich zu einem rechtlichen Problem in umgekehrter Richtung werden: Am Ende verklagt noch jemand (z.B. die Europäische Kommission, der Rat, das EP oder die EZB selbst) zum Beispiel Deutschland, weil Forderungen staatlicher Stellen (es gab da kürzlich recht harsche Forderungen des hessischen Justizministers an die EZB) gegen Art. 130 AEUV verstoßen ...

VI. Unabhängigkeit heißt aber nicht, dass man tun kann, was man will. Der EuGH hat im Juli 2003 klargestellt, dass die EZB der Kontrolle durch den EuGH unterliegt. In dem Urteil heißt es wörtlich (Rs. C-11/00, u.a. Leitsatz 6):

\begin{abstract}
„Dagegen hat diese Zuerkennung einer solchen Unabhängigkeit nicht zur Folge, dass die Europäische Zentralbank völlig von der Europäischen Gemeinschaft gesondert und von jeder Bestimmung des Gemeinschaftsrechts ausgenommen wäre. Nichts erlaubt es, von vornherein auszuschließen, dass der Gemeinschaftsgesetzgeber gemäß den Befugnissen, über die er nach dem EG-Vertrag verfügt, und unter den im EG-Vertrag vorgesehenen Bedingungen normative Maßnahmen erlässt, die Geltung gegenüber der Europäischen Zentralbank beanspruchen können."
\end{abstract}

Klingt an sich unmissverständlich: Die EZB steht ganz eindeutig nicht außerhalb des Rechts, noch nicht einmal außerhalb des Sekundärrechts. Das heißt aber noch nicht, dass der EuGH die EZB auch immer und stets sehr eng kontrollieren würde. Viel wahrscheinlicher ist er ihr einen weiten Spielraum einräumt, um zu entscheiden, was zu tun ist, um die Währung stabil zu halten. Das wäre jedenfalls eine sinnvolle Rechtsprechung im Sinne richterlicher Zurückhaltung im Wissen um die Grenzen dessen, was ein Gericht in der Euro-Krise leisten kann. Dass der EuGH die Lage besser überblickt als die EZB, ist jedenfalls nicht wahrscheinlich. Das gilt übrigens auch für das BVerfG im Verhältnis zu den anderen Akteuren in der Euro-Krise.

Teil 2 folgt!

LICENSED UNDER CC BY NC ND

SUGGESTED CITATION Mayer, Franz C.: Die EZB vor Gericht? Teil I: Wer klagen könnte, und wogegen, VerfBlog, 2012/9/10, http://verfassungsblog.de/die-ezb-vor-gericht-teil-wer-klagen-knnte-und-wogegen/. 